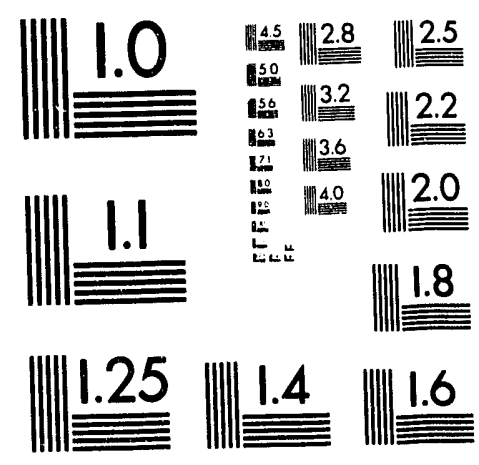



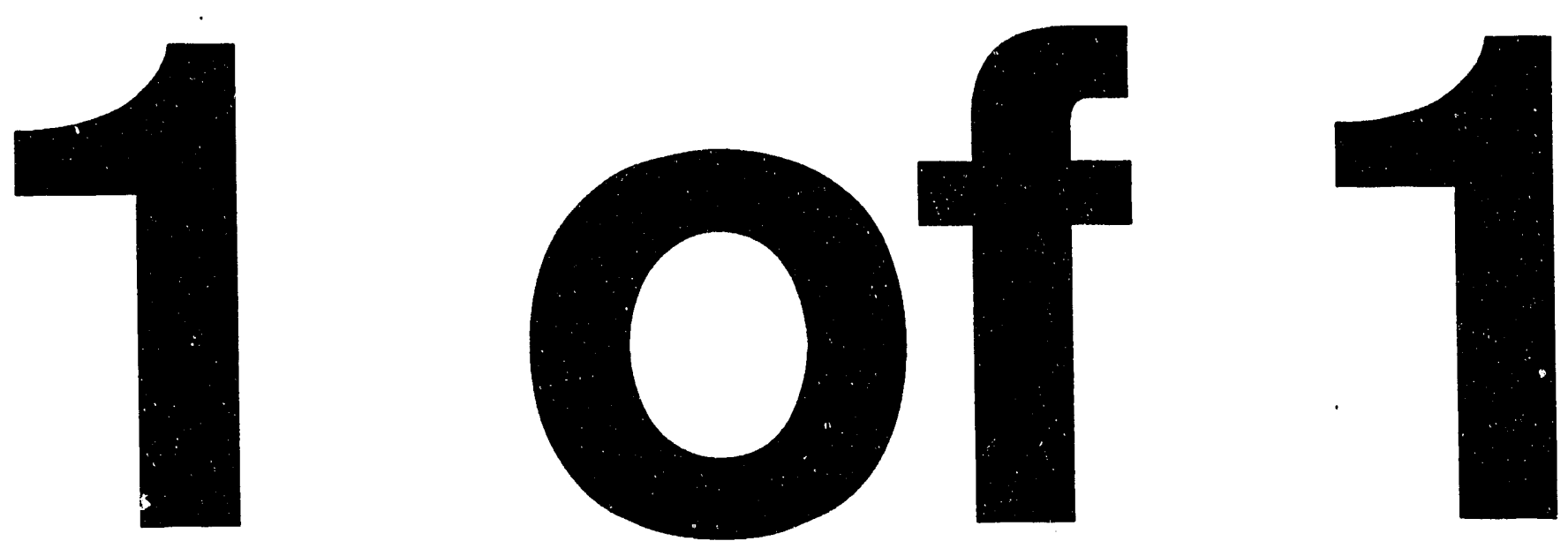


\title{
Simulation of the ALS Longitudinal Multibunch Feedback System
}

\author{
J. Byrd \\ Accelerator and Fusion Research Division \\ Lawrence Berkeley Laboratory \\ University of California \\ Berkeley, CA 94720
}

May 1993

This work was supported by the Director, Office of Energy Research, Office of Basic Energy Sciences, Materials Sciences Division, of the U.S. Department of Energy under Contract No. DE-AC03-76SF00098. 


\title{
Simulation of the ALS Longitudinal Multibunch Feedback System*
}

\author{
John Byrd \\ Lawrence Berkeley Laboratory \\ Berkeley, CA 94720 USA
}

\section{Abstract}

Longitudinal coupled bunch growth rates in the Advanced Light Source (ALS), a $1.5 \mathrm{GeV}$ electron storage ring for producing synchrotron radiation, indicate the need for damping via a feedback (FB) system. The design of the system is based on the proposed PEP-II longitudinal FB system which uses a digital filter to provide the required phase and amplitude response. We report the results of a detailed computer simulation of the FB system including single particle longitudinal beam dynamics, measured RF cavity fundamental and higher order modes, and response of major FB components such as the power amplifier and kicker. The simulation addresses issues such as required FB power and gain, noise, digital filter effects, and varying initial bunch conditions.

\section{INTRODUCTION}

The calculated longitudinal multibunch growth times resulting from interactions with the RF cavity higher order mode impedance[1] indicate the need for a multibunch FB system in order to maintain longitudinal beam stability. The longitudinal feedback (LFB) system proposed for the ALS is based on a design for the FB system proposed for PEP-II, a high-current, many-bunch storage ring proposed to be built a SLAC[2].

A time-domain computer simulation of the longitudinal motion of a multibunch beam[3] has been used in order to study the performance of the ALS LFB system under a variety of conditions. The simulation is useful for studying many issues which theoretical methods on coupledbunch beam stability can only estimate. These include transients and growth rates for nonuniform bunch filling patterns, residual beam noise, nonlinearities, and power requirements. The simulation is also useful for examining electronic effects which can occur in the FB system such as amplifier saturation, limited frequency response, deviations from linear phase.

\section{Longitudinal Sim Ulation[3]}

\section{Difference Equations}

The simulation code models each bunch as a single macroparticle of charge $q$. The coordinates used for describing the longitudinal motion of each bunch are $\Delta E$, the energy deviation from the reference energy, and $\phi$, the phase of the RF clock at the arrival time of the bunch at the cavity.

* This work was supported by the Director, Office of Energy Research, Office of Basic Energy Sciences, Materials Sciences Division, of the U.S. Department of Energy under Contract No. DE-ACO376SF00098.
The turn-by-turn difference equations for these coordinates can be written as

$$
\Delta E_{i+1}=\Delta E_{i}+q V_{g} \sin \phi_{i}-U_{s, i}+q V_{w}+q V_{F B}+q V_{\text {noise }}
$$

and

$$
\phi_{i+1}=\phi_{i}+2 \pi \alpha h \frac{\Delta E_{i}}{E} .
$$

$V_{g}$ is the voltage in the cavity from an external generator, $V_{w}$ is the wakefield voltage, $V_{F B}$ is the voltage kick from the FB system, and $V_{\text {noise }}$ is a noise source. $U_{s, i}$ is the energy lost to synchrotron radiation on turn $i$ and is given by

$$
U_{s, i}=U_{0}+\Delta E_{i} \frac{\partial U}{\partial E}
$$

where $U=U(E)$ is the energy lost to radiation for an electron of energy $E$.

To include effects from the fundamental mode such as Robinson damping and beam gap transients, it is necessary to apply standard beam-loading compensation to the RF voltage[4]. To do this, the fundamental mode is detuned from the RF frequency and $V_{g}$ is adjusted to maintain a constant cavity voltage $\left(V_{c}\right)$. The amount of frequency detuning is given by the relation

$$
\Delta f_{c}=\frac{I_{0} \cos \phi_{s}}{V_{c}}\left(\frac{R}{Q}\right) f_{r f} .
$$

Including beam-loading compensation, Eq. 1 is modified as

$$
\Delta E_{i+1}=\Delta E_{i}+q V_{g} \sin \left(\phi_{i}+\psi\right)-U_{s, i}+q V_{w}+q V_{F B} .
$$

The generator voltage is given by

$$
V_{g}=\left(V_{c}+V_{b r}\right) \cos \psi
$$

where

$$
V_{b r}=\frac{2 I_{0} R_{3}}{1+\beta}
$$

and the tuning angle, $\psi$, is given by

$$
\tan \psi=\frac{2 Q_{0}}{1+\beta} \frac{\Delta f_{c}}{f_{r f}}
$$

The beam-induced voltage in the fundamental mode is included in the wake voltage, $V_{w}$. It is initialized to its expected steady-state value at the start of a run in order to avoid transient problems.

The wakefield voltage is found by summing the voltage contribution from each cavity mode. Aill cavity modes are 
assumed to be resonator-type impedances. The net wake voltage is given by

$$
V_{w}(t)=\sum_{j=1}^{M} 2 k_{j} q \cos \left(\omega_{r, j} t\right) e^{-\frac{\omega r, j}{2 Q} t}
$$

where $\omega_{r}, Q, k$ are the resonator frequency, quality factor, and loss factor. The loss factor is related to the shunt impedance by

$$
k=\frac{\omega_{r} R_{s}}{2 Q} e^{-\left(\omega_{r} \sigma_{r}\right)^{2}}
$$

Each resonator wake voltage is initialized to zero at the start of the simulation except for the fundamental mode.

\section{Feedback Model[3]}

The purpose of the LFB system is to process a measured bunch phase error and produce an energy correction with a $90^{\circ}$ phase shift at the synchrotron frequency. In the proposed design of the system, a beam phase oscillations are detected at $6 \times f_{r_{f}}$. This signal is digitized and a farm of digital signal processors (DSPs) computes an output using a digital filter with the desired frequency and phase response using a digital filter algorithm. The computed correction is then converted to a voltage and modulated to the operating frequency of the longitudinal kicker.

The digital filter used in the DSPs is a 5-tap finite impulse response (FIR) filter. To reduce the total amount of data processing, only the signal from every $n$th turn for a particular bunch is used to calculate the output. Because of the many $(\sim 110)$ turns per synchrotron oscillation, a reduced set of sampled signals is adequate to define the synchrotron oscillation for a given bunch. On turns where a new output for a given bunch is not calculated, the previous correction for that bunch is used. For the simulation, a downsampling factor of $n=24$ was used, corresponding to roughly 5 samples per synchrotron oscillation.

The correction signal is calculated from the input phase error signal as a convolution given by

$$
Y_{k}=\sum_{n=0}^{m-1} C_{n} X_{k-n}
$$

where $Y_{k}$ is the output of the filter on turn $k, n$ is an index which sums over the last $m$ phase error measurements of a particular bunch, $C_{n}$ are the weighting coefficients of the filter, and $X_{k-n}$ are the digitized input phase error measurements for the last $m$ samples. The filter coefficients are chosen to pass signals at the synchrotron frequency with a $90^{\circ}$ net phase shift and zero DC response. The filter coefficients for one of the 5-tap filters used in this simulation and the resulting frequency response is shown in Figure 1.

The simulation LFB model includes: 1) the properties of the phase detector, mixer, low-pass filter, low-pass filter, $A / D$ and $D / A$ conversion, 2) input noise, gain, and ifset errors, 3) algorithm running DSPs to compute output response, 4) bandwidth limitations of amplifier, and kicker.

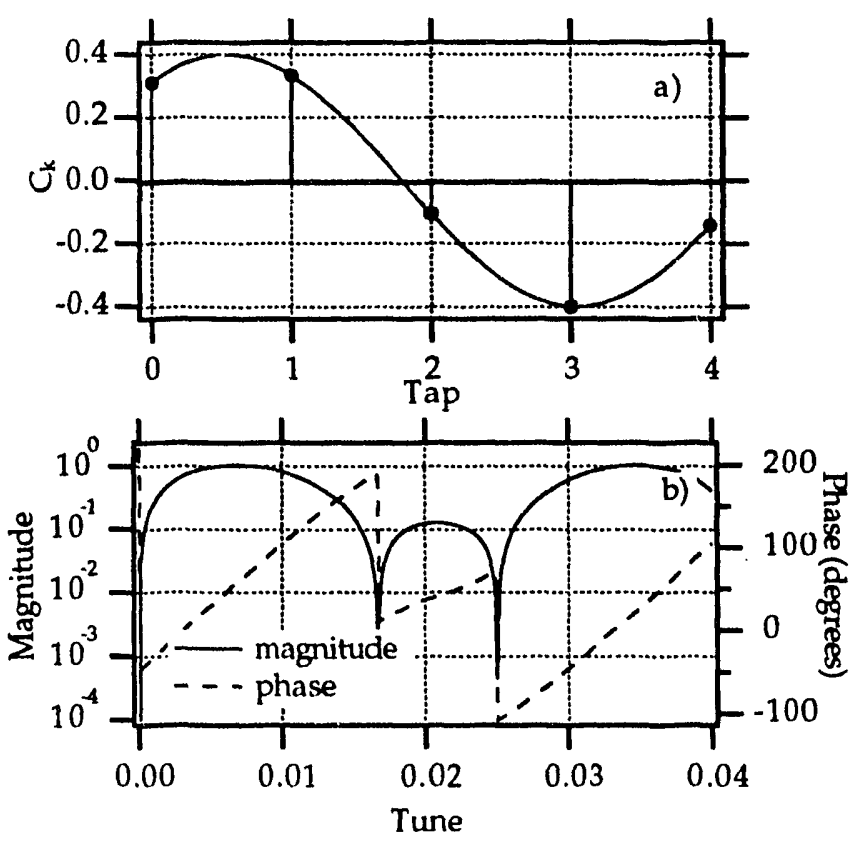

Figure 1: a) Filter coeffecients used in FIR filter. b) Frequency response of FIR filter.

Digital filters were used to approximate the frequency response of the LFB system components in the simulation. A wide variety of filters were tried. For the results in this paper, a 20-tap FIR bandpass filter was used to approximate frequency response of the analog components in the system.

\section{RESUlts}

The ALS and FB parameters used for the simulation are shown in Table 1. The monopole RF cavity modes used were measured on a spare ALS cavity and are given in reference[5], in these proceedings. One of the consequences of the gap in the bunch filling pattern is a different synchronous phase angle for each bunch. This makes the zero

\begin{tabular}{clc}
\hline \hline Parm. & Description & Value \\
\hline$E_{b}$ & Beam energy & $1.5 \mathrm{GeV}$ \\
$C$ & Circumference & $196.8 \mathrm{~m}$ \\
$h$ & Harmonic number & 328 \\
$N$ & Number of bunches & 280 \\
$I_{0}$ & Total DC beam current & $0.4 \mathrm{~A}$ \\
$Q_{s}$ & Synchrotron Tune & 0.009 \\
$\tau_{s}$ & Rad. damping time & $22500 \mathrm{turns}$ \\
$\delta \phi_{i n j}$ & Inj. phase offset & $0.20 \mathrm{rad}$ \\
$(\delta E / E)_{i n j}$ & Inj. energy offset & 0.002 \\
$V_{F B, m a x}$ & Maximum FB kick & $1.5 \mathrm{kV}$ \\
$V_{n o i s e}$ & RMS noise & $50 \mathrm{~V}$ \\
$\tau_{C B}$ & Max. CB growth time & $1500 \mathrm{turns}$ \\
$\tau_{F B}$ & Max. FB damping time & $600 \mathrm{turns}$ \\
\hline \hline
\end{tabular}

Table 1: ALS and LFB parameters used in the simulation. 


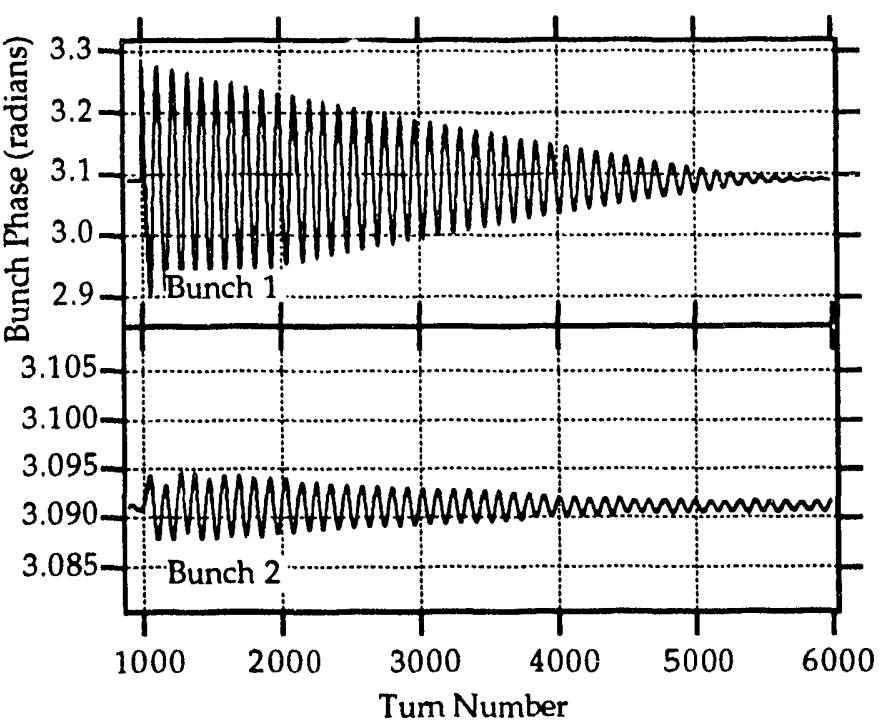

Figure 2: Transient response of two of the 280 bunches with feedback.

DC response of the FIR filter important. Since all bunches start at the same initial phase, the simulation is run for 1000 turns before an injection disturbance is applied in order to allow the beam to settle into its steady-state condition.

All simulation runs used ALS injection conditions. The injector system injects 4 bunches separated by 4 RF buckets into the storage ring at a $1 \mathrm{~Hz}$ rate. Each bunch has a maximum centroid energy and phase offset given in Table 1. Runs with the FB turned off show an exponential increase in the bunch phase up to an amplitude of $\sim 0.3$ rad whereupon the oscillation appears to self-limit. The limiting mechanism is not yet understood. The transient responses of an offset bunch (bunch 1) and a trailing bunch (bunch 2) with the FB on are shown in Figure 2. Bunch 1 is kicked at turn 1000 and is damped linearly while the FB voltage is saturated at $1.5 \mathrm{kV}$ and exponentially in the proportional region. Bunch 2 is excited through wakefield coupling and is damped down to the sensitivity level of the FB input, which is determined by the least significant bit of the input $A / D$ conversion. For the simulation, the sensitivity was $2 \mathrm{mrad}$.

Shown in Figure 3 is an expanded view of the bunch phase and the computed FB output kick for bunch 1 . The stepping structure of the output is a result of the downsampling of the input signal. Note that the output is shifted by $\sim 90^{\circ}$ from the bunch phase and that the output has no DC component. The damping rate of the LFB system was set to be 2.5 times the largest calculated $C B$ growth rate.

For the FB gain and external noise used, the system was always capable of damping the bunch phases to within the sensitivity level of FB input. The requisite voltage to damp the beam was also studied. The FB system was able to stabilize the beam for the above injection transients with $\sim 1 / 2$ the maximum FB voltage kick. Thus, the system could concievable run with $\sim 1 / 4$ the specified power for

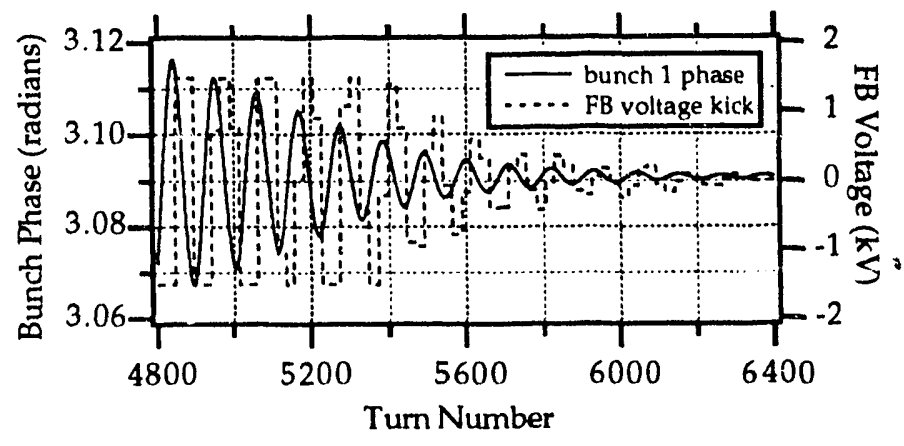

Figure 3: Transient response of bunch 1 and the FB output. The FB is saturated up to turn 4700 .

the kind of injection disturbances expected

The effect of shifts in the HOM frequencies on the ability of the FB system to damp the beam was also studied. We ran a series of simulation runs using the measured HOM parameters, where several of the high- $Q$ HOM frequencies were varied randomly from run to run. The FB was able to damp the beam under all conditions except when the frequency strongest HOM landed on a frequency corresponding to an unstable coupled-bunch mode.

\section{CONCLUSIONS}

A simulation of the ALS longitudinal multibunch FB system has demonstrated that it is capable of damping coupled-bunch oscillations to acceptable levels for nominal operating conditions. The actual power requirements should be less than calculated.

The author would like to thank Kathy Thompson for providing the original multibunch simulation code and Don Briggs, Walid Hosseini, Haitham Hindi, and John Fox for providing the code for the FB model. Thanks also to Glen Lambertson for many useful discussions, Johan Bengtsson for assistance in deciphering the simulation code, and to members of the Center for Beam Physics at LBL for their general support and encouragement.

\section{REFERENCES}

[1] J. Byrd and J. Corlett, Study of Coupled-bunch Collective Effects in the $A L S$, these proceedings.

[2] Design Update for PEP-II, LBL PUB-5303 (1992).

[3] D. Briggs et. al., Computer Modelling of bunch-bybunch feedback for the SLAC B-factory design, Proceedings of the 1991 IEEE Particle Accelerator Conf. (1991).

[4] See, for example, P. Wilson, Proceedings of the 1982 Particle Accelerator School, AIP Conf. Proc. 127 (AIP, New York 1982).

[5] J. Corlett, J. Byrd, Measurement of Higher-order Modes of the ALS $500 \mathrm{MHz}$ Accelerating Cavities, these proceedings. 

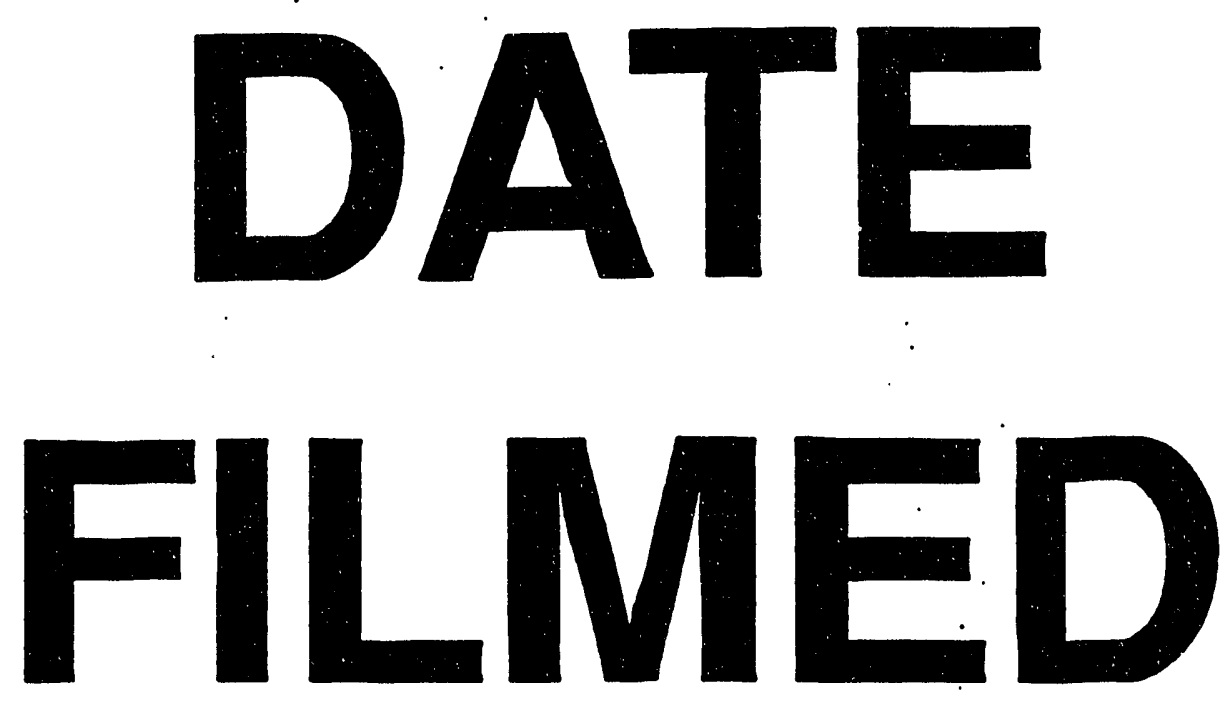

$10 / 19 / 93$
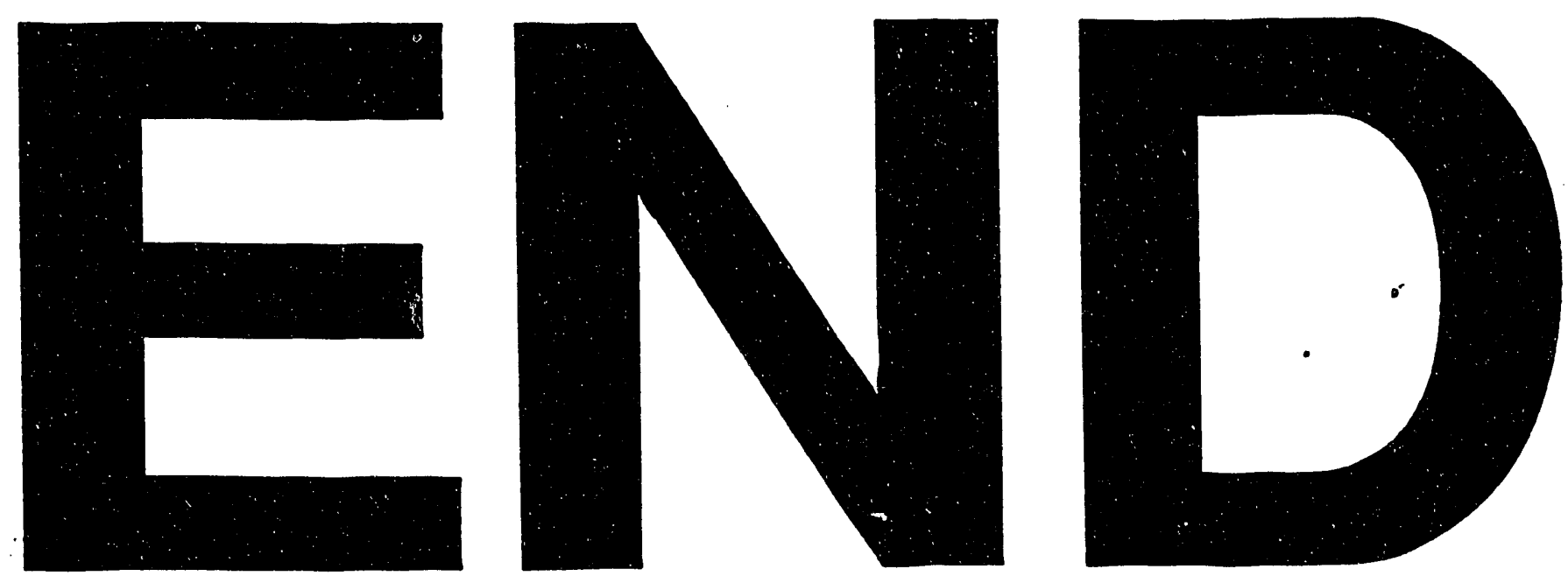
